\title{
Master-slave manipulator for laparoscopic surgery using a 6-axis vertical articulated robot
}

\author{
Makoto Jinno
}

\begin{abstract}
Laparoscopic surgery is a minimally invasive surgery that accelerates postoperative recovery, but it can only be performed by surgeons with advanced surgical skills. One of the main difficulties in laparoscopic surgery is restriction of free motion of the forceps because of limited degrees of freedom by the trocar. Recently, many master-slave manipulators with a remote center-of-motion mechanism have been used in laparoscopic surgery to solve this problem.

The master-slave manipulator for laparoscopic surgery using a 6-axis vertical articulated robot has some advantages as a scalable and versatile system. However, to achieve smooth insertion and removal motion of the forceps from the trocar, the problem of restriction of motion by the trocar must be addressed.

This paper describes a master-slave manipulator system for laparoscopic surgery using a 6-axis vertical articulated robot with a slave arm containing forceps. A manipulating mode of the slave arm, consisting of four basic motion modes and three control modes, is proposed in order to perform smooth operations when the motion of the trocar is restricted. The three control modes include a non-trocar mode, a trocar mode, and a transitional mode between the two modes in order to ensure smooth insertion and removal motions of the forceps. Furthermore, the transitional mode consists of a posture adjustment motion and insertion-removal motions. The posture adjustment motion before an insertion motion of the forceps under the transitional mode is achieved by the motions of only the 4th axis and 5th axis of the slave arm. The posture adjustment motion and insertion motion under the transitional mode and the master-slave motion under the trocar mode are shown through evaluations of the master-slave manipulator system, including a 6-axis vertical articulated robot, in order to verify the effectiveness of the proposed methods.
\end{abstract}

Keywords: Surgical robotics; Minimally invasive surgery; Master-slave manipulator; Vertical articulated robot; Trocar; Forceps

\section{Background}

Laparoscopic surgery is one of the most common minimally invasive surgical strategies. As shown in Figure 1 depicting conventional laparoscopic surgery, a surgeon performs procedures using forceps passed through trocars (each approximately 5 to $10 \mathrm{~mm}$ in diameter) into a patient's abdominal cavity while watching an image of the abdominal cavity acquired by a laparoscope. With a small incision, the patient can recover sooner and medical costs are reduced. Therefore, laparoscopic surgery is highly advantageous for the patient and has become widespread in recent years [1].

However, laparoscopic surgery can only be performed by surgeons with advanced surgical skills. One of the main difficulties in laparoscopic surgery is a restriction

Correspondence: Makoto_Jinno@terumo.co.jp

Terumo Corporation, Nakai-machi, Ashigarakami-gun, Kanagawa 259-0151, Japan

\section{Springer}

(C) 2014 Jinno; licensee Springer. This is an Open Access article distributed under the terms of the Creative Commons Attribution License (http://creativecommons.org/licenses/by/4.0), which permits unrestricted use, distribution, and reproduction in any medium, provided the original work is properly credited. of the free motion of the forceps because of lack of degrees of freedom (DOF) by the trocar. The position and posture of the gripper of the forceps cannot be changed freely in the patient's abdominal cavity during the procedure.

Recently, many master-slave manipulators have been used to solve the difficulties in laparoscopic surgery [2]. The surgeon manipulates the master arm. The slave arm motion is controlled at the same or reduced scale of motion of the master arm. The slave arm has a remote center-ofmotion (RCM) mechanism to enable performance of surgical procedures under restricted motion of the trocar [3-5].

Various studies have reported master-slave manipulators for laparoscopic surgery using 6-axis vertical articulated robots or 7-axis redundant vertical articulated robots [6-8]. Furthermore, support mechanisms have been reported for a robotic forceps using a 6-axis articulated robot with active and passive joints [9]. The master-slave manipulator 


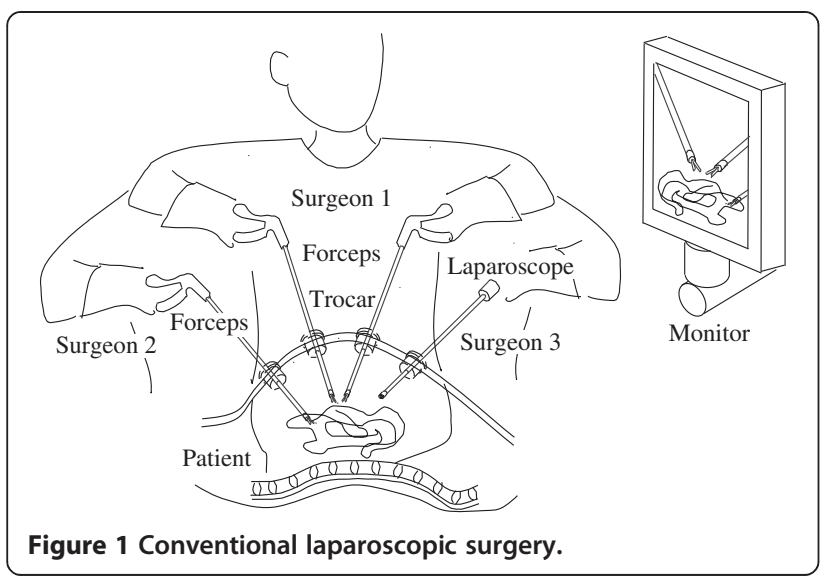

for laparoscopic surgery using a 6-axis vertical articulated robot is advantageous as it is a scalable and versatile system. However, to achieve smooth insertion and removal motion of the forceps from the trocar, the problem of the restricted trocar movement must be addressed.

This paper first presents a comparison between 6-axis vertical articulated robots and RCM robots, along with the kinematics of a trocar mode. Second, a manipulating mode of the slave arm consisting of four basic motion modes and three control modes is proposed in order to perform smooth operations under restricted trocar movement. Third, the transitional mode, which is one of three control modes, and a posture adjustment motion before insertion of the forceps under the transitional mode are proposed. In addition, the transition state between each control mode is examined. Fourth, an evaluation experimental system, which consists of a slave arm, a master arm, and a controller, is presented. Finally, experimental results are presented to confirm the effectiveness of the basic motion modes, and the methods used in the transition mode between non-trocar and trocar modes are reported.

\section{Methods}

\section{Comparison between RCM robots and 6-axis vertical articulated robots}

The master-slave manipulator used for laparoscopic surgery in clinical practice has an RCM mechanism to solve the problem of restricted trocar movement. The RCM mechanism has a parallel link mechanism or circular guide mechanism to achieve in/out, up/down, and right/left motions of the forceps in the patient's abdominal cavity. Therefore, the installation position of the base frame of the slave arm is determined based on the trocar position in order to be at the same position as the RCM. The slave arm has 7 DOF; the configuration is $3 \mathrm{DOF}$ of the RCM mechanism for positioning the gripper, 3 DOF on the tip of the slave arm for posture adjustment of the gripper, and 1 DOF for grasping by the gripper. The slave arm can perform grasping, dissecting, suturing, ligaturing, and cutting tasks from any position and posture inside the patient's abdominal cavity. The 3 DOF on the tip of the slave arm consist of a roll axis formed by rotation of the forceps shaft, a pitch and yaw axis, and a yaw and roll axis.

In the case of a 6-axis vertical articulated robot for slave arm, the RCM can be controlled by inverse kinematics under conditions of movement restriction by the trocar. The control mode is defined as the trocar mode in this paper.

The entry point into the patient's abdominal cavity is located at any position in the working area of the slave arm. Therefore, it is possible to insert forceps through the other trocar in a different position without changing the base frame position of the slave arm. The working area of the forceps in the patient's abdominal cavity depends on the relationship between trocar position and base frame position of the slave arm.

The 6-axis vertical articulated robot has a wide working area outside the patient's abdominal cavity. Therefore, parts of motions can be automated, such as insertion motion, removal motion, exchange motion to the other forceps, and movement to the home position away from the patient.

Furthermore, the master-slave manipulator using a 6-axis vertical articulated robot is expected to be a versatile system that can be used as a laparoscopeholding robot, conventional forceps-holding robot, robotic forceps-holding robot, assistant surgeon robot, and open surgery robot. Furthermore, the master-slave manipulator is a scalable system, from a single-slave-arm system to a multi-slave-arm system.

\section{Kinematics of trocar mode}

Figure 2 shows the joint configuration of the slave arm consisting of a 6-axis vertical articulated robot and an articulated forceps. When using a 6 -axis vertical articulated robot, the slave arm requires 9 DOF including a gripper in order to move the tip of the forceps to any position and posture within the patient's abdominal cavity. This is because the DOF are restricted due to the use of a trocar. In the case of motion only inside the patient's abdominal cavity, 2 DOF of the wrist joint of the 6-axis vertical articulated robot (4th axis and 5th axis) are used as a passive joint. However, every joint must be active to manipulate the forceps in any position and posture outside the patient's abdominal cavity.

Furthermore, in this paper, 3 conditions are proposed:

(1) The 4th, 5th, and 6th axes intersect at a point. These joints are called manipulator wrist joint coordinate systems. The manipulator wrist joint coordinate systems are the same as those found in conventional industrial robots. 


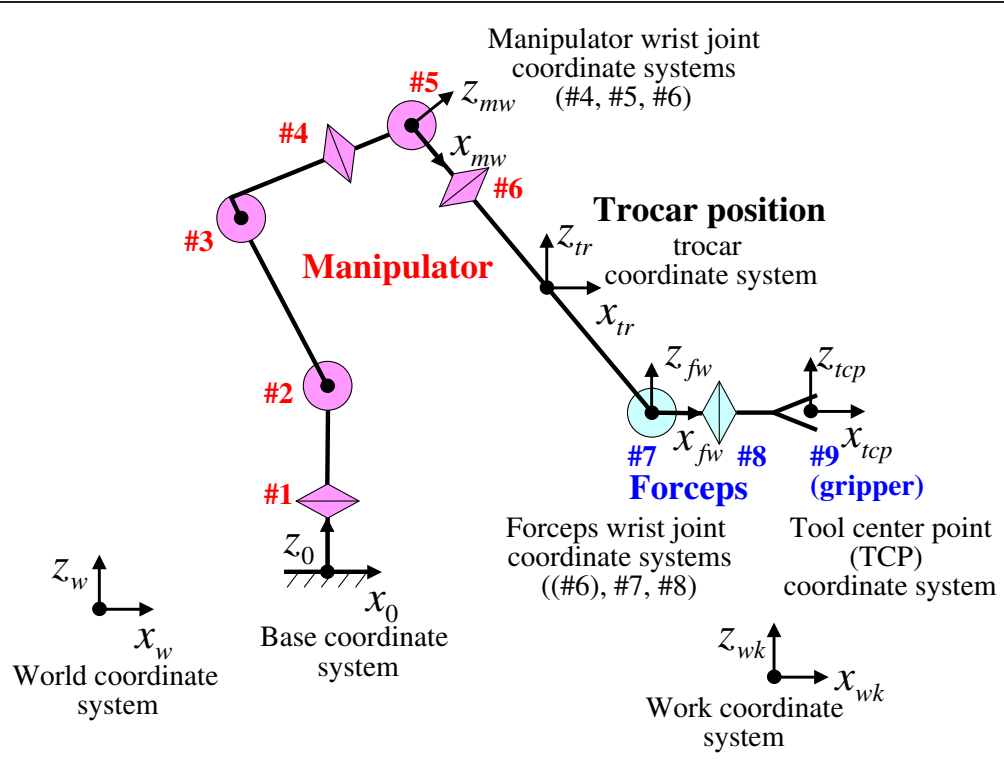

Figure 2 Joint configuration of slave manipulator.

(2) The 6th axis accords with the forceps axis. Rotation of the forceps axis can be performed by rotation of only the 6th axis.

(3) The 7th and 8th axes are located at the distal end of the forceps, and intersect the 6th axis at a point. These joints are called forceps wrist joint coordinate systems.

The procedure for calculating inverse kinematics of the trocar mode is described in the following 5 steps, as shown in Figure 3:

(1) The position of the forceps wrist joint coordinate systems is calculated from the position and posture of the tool center point.

(2) The position of the manipulator wrist joint coordinate systems is calculated from the position of the forceps wrist joint coordinate systems and the trocar position.

(3) The joint angles of the 1st, 2nd, and 3rd axes are calculated from the position of the manipulator wrist joint coordinate systems.

(4) The joint angles of the 4th and 5th axes are calculated from the trocar position and the joint angles from the 1st axis to the 3rd axis.

(5) The joint angles of the 6th, 7th, and 8th axes are calculated from the position and posture of the tool center point and the joint angles from the 1st axis to the 5 th axis.

In this manner, the solution of each joint angle is obtained.
In the following section, the slave arm is examined without the 7th and 8th axes at the tip of the forceps to discuss the basic characteristics of using a 6-axis vertical articulated robot for the slave arm. The slave arm without the 7 th and 8th axes at the tip is the same configuration as that combined with a laparoscope or conventional forceps instead of articulated forceps.

\section{Basic motion mode}

Different types of motion modes are required inside and outside the patient's abdominal cavity, depending on the

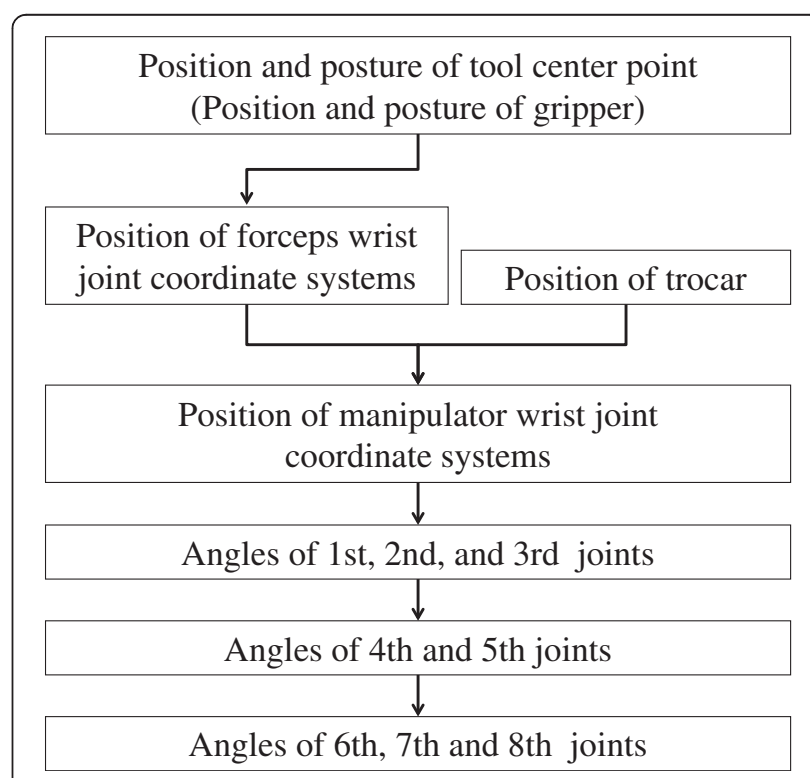

Figure 3 Calculating flow of inverse kinematics of trocar mode. 


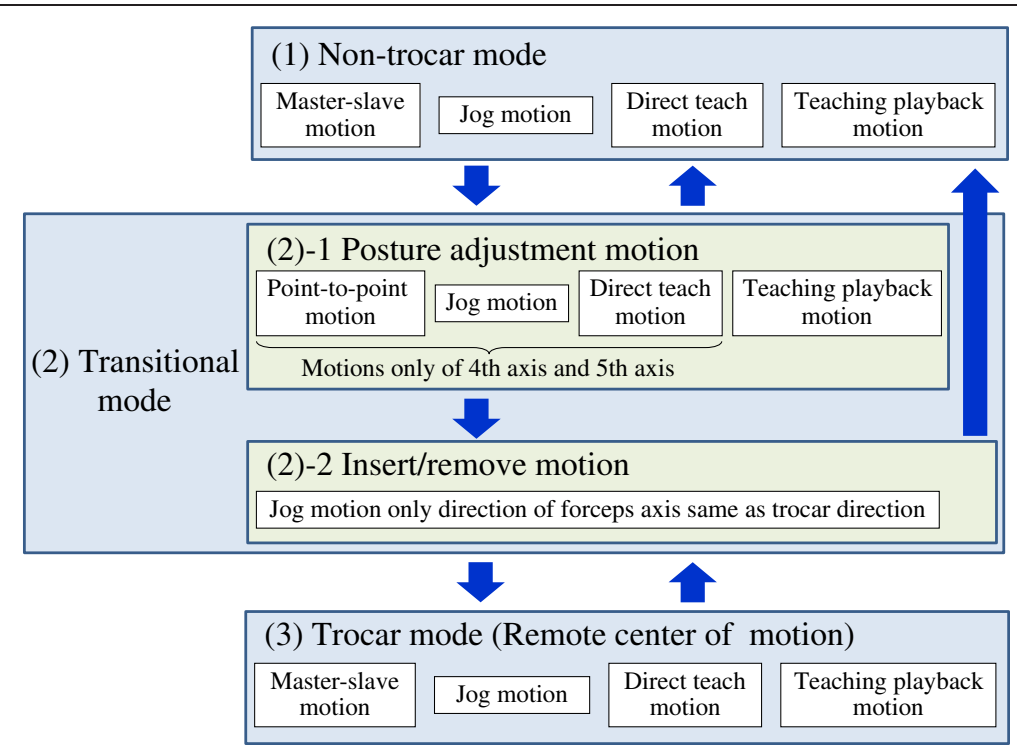

Figure 4 Control mode and state transition.

various situations of laparoscopic surgery. Four basic motion modes for manipulation of the slave arm are proposed:

\section{Master-slave motion}

The master arm manipulates the slave arm. The master-slave motion is the most basic motion to manipulate the forceps inside the patient's abdominal cavity for an operation. Intuitive hand-eye coordination is obtained by adequate definition of various coordinate systems. For example, coordinate systems might include the world coordinate system, base coordinate system of the slave arm and the master arm, and work coordinate system, depending on the slave arm position and line of sight from the laparoscope.

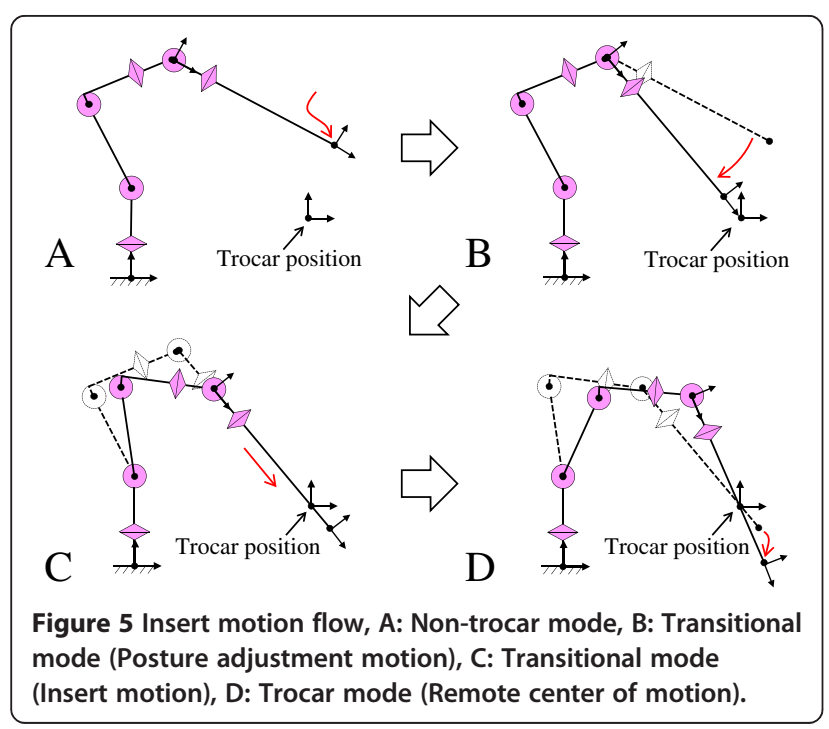

\section{Jog motion}

The tip of the forceps is moved by the user interface, a teaching pendant, or an operating panel at a fixed, prescribed speed for each direction of the coordinate axis in the world, base, work, forceps, and joint coordinate systems. The jog motion is used to achieve precise linear motion and positioning that are difficult to obtain with a master-slave motion.

\section{Direct teach motion}

In the case of the direct teach motion, the slave arm is moved by the operator's hands to drive the robot arm joint from the output side without using a human-machine interface such as the master arm or a teaching pendant. The operator can perform manipulations at the patient's bedside while watching the configuration of the slave arm. Direct teach motion is achieved by compensation control of coulomb and viscous friction of each joint and the weight torque of the 2 nd and 3rd axes. The desired angles of the 4th and 5th axes under the trocar mode are calculated by the position of the manipulator wrist joint coordinate systems. The position of the manipulator wrist joint coordinate systems is calculated by the current angles of the 1st, 2nd, and 3rd axes. Therefore, in the trocar mode, the 4th and 5th axes are controlled by conventional position control, whereas the 1st, 2nd, and 3rd axes are controlled by the direct teach motion.

\section{Teaching playback motion}

The teaching playback motion is an automatic motion based on the teaching point data and motion programs. The teaching playback motion is used mainly for structured tasks such as the forceps change motion or the 


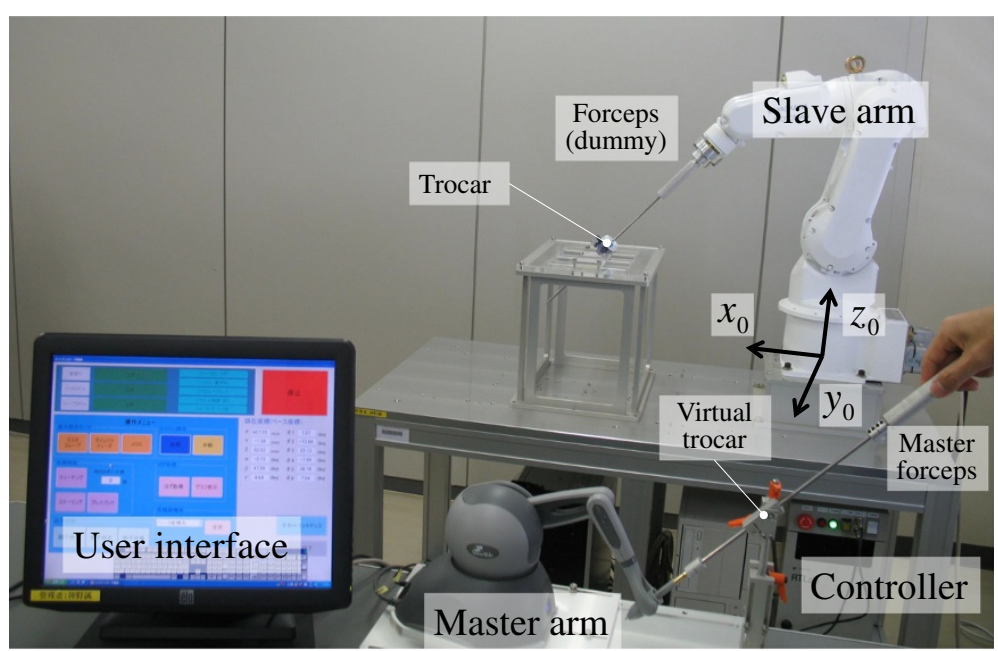

Figure 6 Configuration of evaluation experimental system.

return motion to the home position in order to reduce the surgeon's workload and improve the efficiency of the operative procedure.

When the forceps is manipulated by the basic motion mode in the patient's abdominal cavity, the slave arm must be controlled based on the kinematics of the trocar mode.

\section{Main control mode}

When the forceps is inserted into or removed from the trocar, easy handling is desired without increasing the

Table 1 Specifications of slave arm system

\begin{tabular}{lll}
\hline 6-axis vertical articulated robot (MOTOMAN-MH3F) \\
\hline Payload & $3 \mathrm{~kg}$ \\
Lower arm length & $260 \mathrm{~mm}$ & \\
Upper arm length & $270 \mathrm{~mm}$ (offset $30 \mathrm{~mm}$ ) & \\
Wrist length & $90 \mathrm{~mm}$ (from wrist joint to mechanical interface) \\
Vertical Reach & $804 \mathrm{~mm}$ & \\
Horizontal reach & $532 \mathrm{~mm}$ & Maximum speed \\
Joint & Maximum motion range & $150^{\circ} / \mathrm{s}(3.49 \mathrm{rad} / \mathrm{s})$ \\
1st Axis (Turning) & $-160^{\circ} \sim+160^{\circ}$ & $\mathrm{rad} / \mathrm{s})$ \\
2nd Axis (Lower arm) & $-85^{\circ} \sim+90^{\circ}$ & $190^{\circ} / \mathrm{s}(3.32 \mathrm{rad} / \mathrm{s})$ \\
3rd Axis (Upper arm) & $-105^{\circ} \sim+260^{\circ}$ & $300^{\circ} / \mathrm{s}(5.24 \mathrm{rad} / \mathrm{s})$ \\
4th Axis (Wrist roll) & $-170^{\circ} \sim+170^{\circ}$ & $300^{\circ} / \mathrm{s}(5.24 \mathrm{rad} / \mathrm{s})$ \\
5th Axis (Wrist pitch) & $-120^{\circ} \sim+120^{\circ}$ & $420^{\circ} / \mathrm{s}(7.33 \mathrm{rad} / \mathrm{s})$ \\
6th -Axis (Wrist roll) & $-360^{\circ} \sim+360^{\circ}$ & \\
Weight & $27 \mathrm{~kg}$ & \\
\hline Dummy forceps & & \\
\hline Shaft diameter & $\varphi 5 \mathrm{~mm}$ & $350 \mathrm{~mm}$ \\
Shaft length & $500 \mathrm{~mm}($ from mechanical interface to tip) \\
\hline Total length & & \\
\hline
\end{tabular}

load on the surgeon under any operating conditions. When using a 6-axis vertical articulated robot for the slave arm, the control algorithm must be changed to reflect a state of being inside or outside the patient's abdominal cavity because the inverse kinematics are different between the non-trocar mode and trocar mode. Therefore, a transition algorithm between the nontrocar mode and trocar mode is required.

Prior research using 7-axis vertical articulated robots [8] proposed an impedance controller that is configured with zero stiffness in translations and high stiffness in the rotations. Therefore, the forceps can only move unrestricted in translations. The operator can hold the slave arm with his/her hands and guide it through the trocar [10].

In this study, to achieve smooth insertion and removal motion of the forceps from the trocar, 3 types

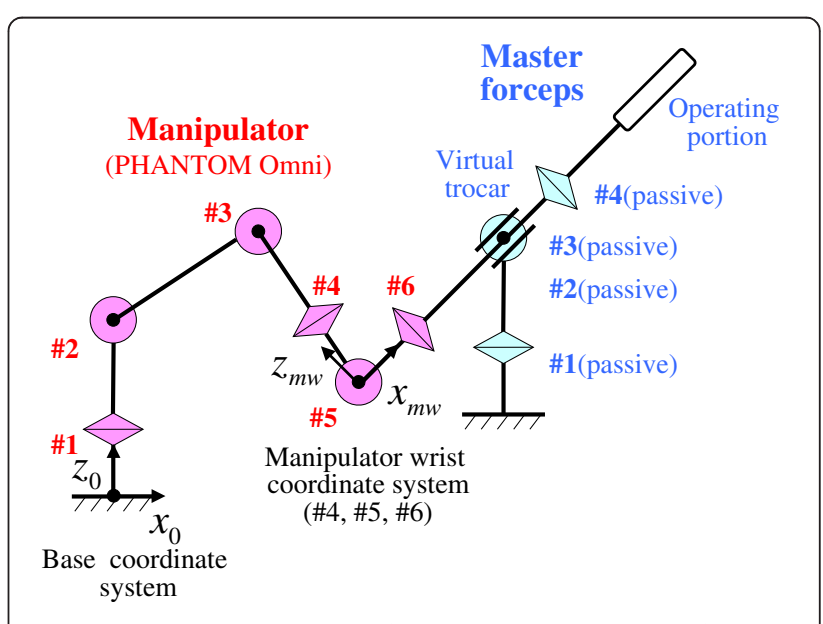

Figure 7 Joint configuration of master arm with dummy forceps. 


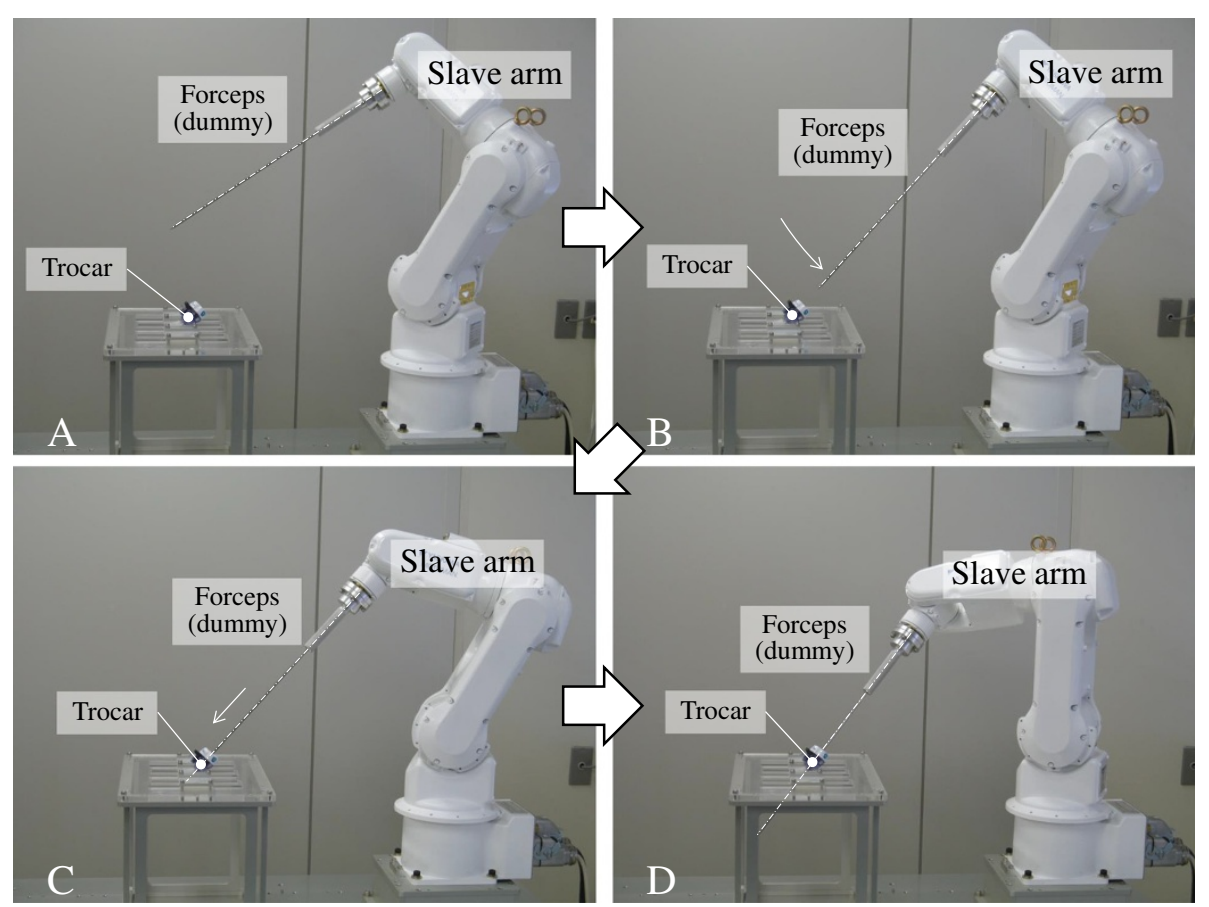

Figure 8 Overview of insert motion, A: Non-trocar mode, B: Transitional mode (Posture adjustment motion), C: Transitional mode (Insert motion), D: Trocar mode (Remote center of motion).

of control modes, including the transition mode, and 4 types of posture adjustment methods are proposed:

\section{Non-trocar mode}

The non-trocar mode is a control mode to manipulate the forceps outside the patient's abdominal cavity. The non-trocar mode uses the conventional inverse kinematics of the 6-axis vertical articulated robot. The joint angles of the forceps wrist joint coordinate systems are the origin angle of each joint.

\section{Transition mode between non-trocar mode and trocar mode}

The transition mode is a control mode to transition from the non-trocar mode to the trocar mode for insertion motion or from the trocar mode to non- trocar mode for the removal motion. The details of the transition mode are described in the next section.

\section{Trocar mode}

The trocar mode is a control mode to manipulate the forceps inside the patient's abdominal cavity. The trocar mode uses the inverse kinematics described in the previous section. Under the non-trocar mode and the trocar mode, it is possible to manipulate the forceps by the 4 types of basic motion modes described in the previous section.
Transition mode between non-trocar mode and trocar mode Furthermore, the transition mode between the non-trocar mode and trocar mode consists of 2 types of motion modes as follows:

\section{Posture adjustment motion}

The posture adjustment motion is the motion to adjust the forceps axis to the direction of the trocar from any position and posture outside the patient's abdominal cavity. The details of the posture adjustment motion are described in the next section. It is necessary to consider the working area of the slave arm and to avoid collision with the trocar or patient.

\section{Insert/remove motion (jog motion only direction of trocar position)}

The insert/remove motion is allowed along the forceps axis only when the forceps axis is in a state that it is equal to the direction of the trocar. It is possible to insert the forceps into the trocar or remove the forceps from the trocar by the jog motion.

This control method must be transferred to the trocar mode via the posture adjustment motion and the insert/ remove motion from the non-trocar mode in order to insert the forceps into the patient's abdominal cavity. The control method can be changed to the trocar mode from the transition mode when the insertion length of the forceps from the trocar position becomes more than $50 \mathrm{~mm}$. 
Furthermore, the control method must be transferred to the non-trocar mode via insert/remove motion from the trocar mode in order to remove the forceps from the patient's abdominal cavity. The control method can be changed to the non-trocar mode from the transition mode when the forceps tip passes through the trocar.

\section{Posture adjustment motion}

It is necessary to obtain an entry point, which is the trocar position for the posture adjustment motion in advance by a teaching function or an image recognition function. The teaching method is as follows.

The entry point is provided by a teaching forceps. The tool center point, which is the teaching forceps tip, is led to the entry point on the patient body by the master-slave motion, jog motion, or direct teach motion. Then, the position is stored as the trocar position. This teaching method is the same as a conventional teaching method of an industrial robot. The desired angles of the posture adjustment motion are calculated from the direction of the trocar position from the origin of the manipulator wrist joint coordinate systems, which is calculated from current angles from the 1st axis to the 3rd axis. As a result, the desired angles of the 4th and 5th axes are provided. Therefore, the posture adjustment motion in order to adjust the forceps axis to the direction of the trocar carries out motion in only the 4th and 5th axes. The posture adjustment motion is chosen from the following 4 types of proposed methods in order to enable effective posture adjustment motion under any operation conditions.

\section{Posture adjustment by point-to-point motion using only the 4th and 5th axes}

The slave arm moves to the desired angles of the 4th and 5 th axes by synchronous motion.

Posture adjustment by jog motion using the 4th and 5th axes The slave arm is moved by jog motion in only one direction to the desired angles of the 4th and 5th axes.

\section{Posture adjustment by direct teach motion using the 4th and 5th axes}

The slave arm is moved by the direct teach motion in only one direction to the desired angles of the 4th and 5 th axes.

\section{Posture adjustment by teaching playback motion}

Posture adjustment by the teaching playback motion is an automatic motion based on the teaching point data file and the motion program file that are made in advance. The forceps axis of the teaching point must be in accordance with the trocar direction. In the case of posture adjustment by teaching playback motion, all joints of the slave arm are moved.

The basic control mode and state transition are shown in Figure 4. The insert motion flow from the non-trocar mode to the trocar mode is shown in Figure 5.

\section{Configuration of evaluation experimental system}

An evaluation experimental system with a 6-axis vertical articulated industrial robot was constructed in order to evaluate the basic motion modes of the kinematics of the trocar mode and the transition algorithm between non-trocar and trocar modes. The evaluation experimental system consisted of a slave arm, a master arm, and a controller as shown in Figure 6.

The 6-axis vertical articulated industrial robot of the slave arm was made by YASKAWA Electric Corporation (MOTOMAN-MH3F) for industrial use. The specification of the slave arm is shown in Table 1. In order to evaluate the positioning of the tip of the forceps without a posture, a dummy forceps with a cylindrical stick shape $5 \mathrm{~mm}$ in diameter was used.

The master arm was a three-dimensional pointing device made by SensAble Technologies, Inc. (PHANTOM Omni). Two types of operating methods of the master

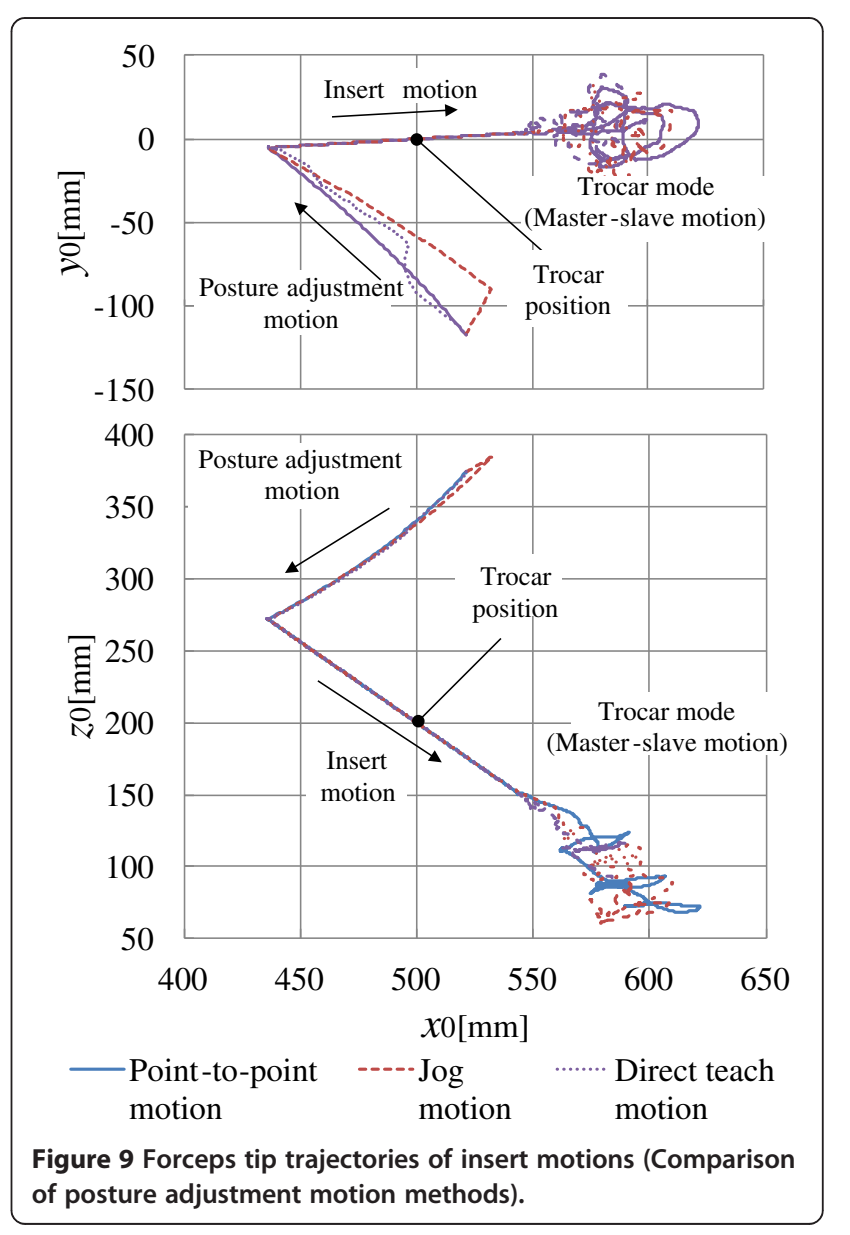




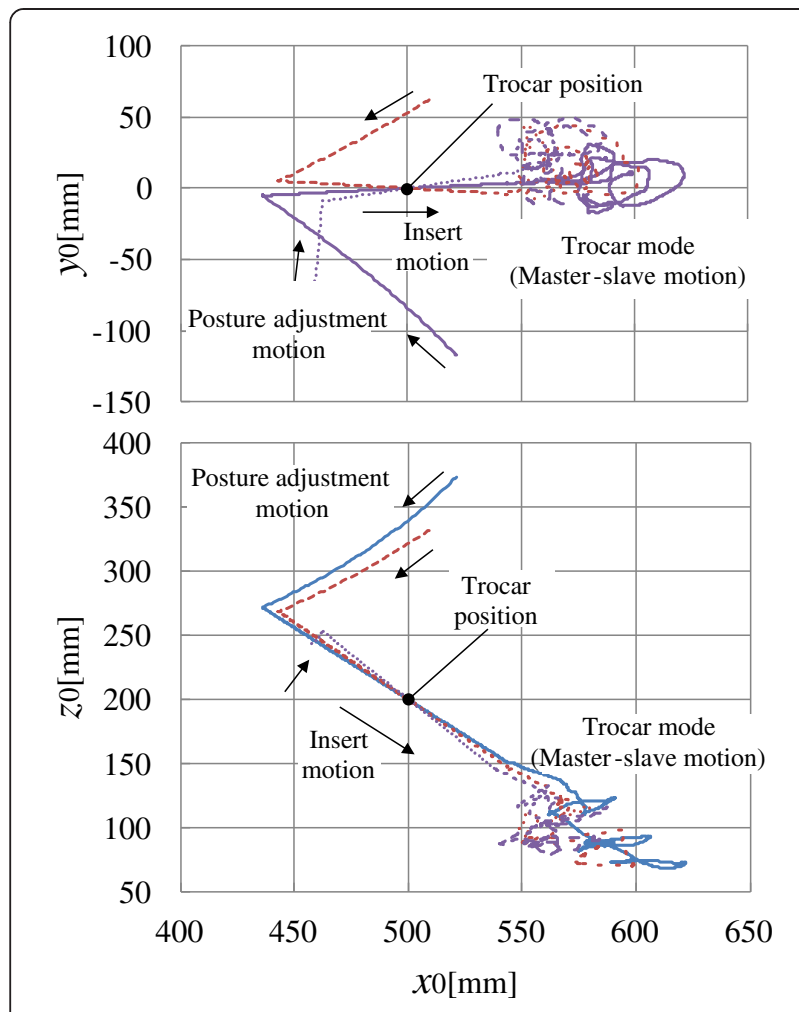

— Initial point 1 -----Initial point 2 ….... Initial point 3

Figure 10 Forceps tip trajectories of insert motions (Comparison of initial positions of forceps tip).

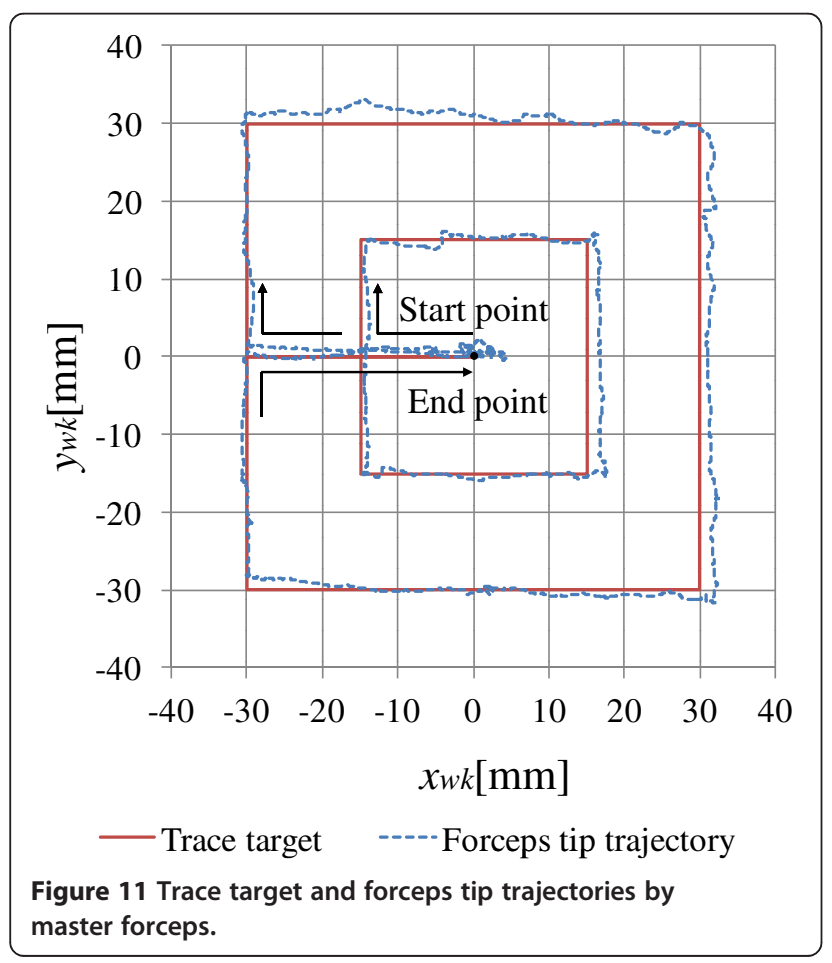

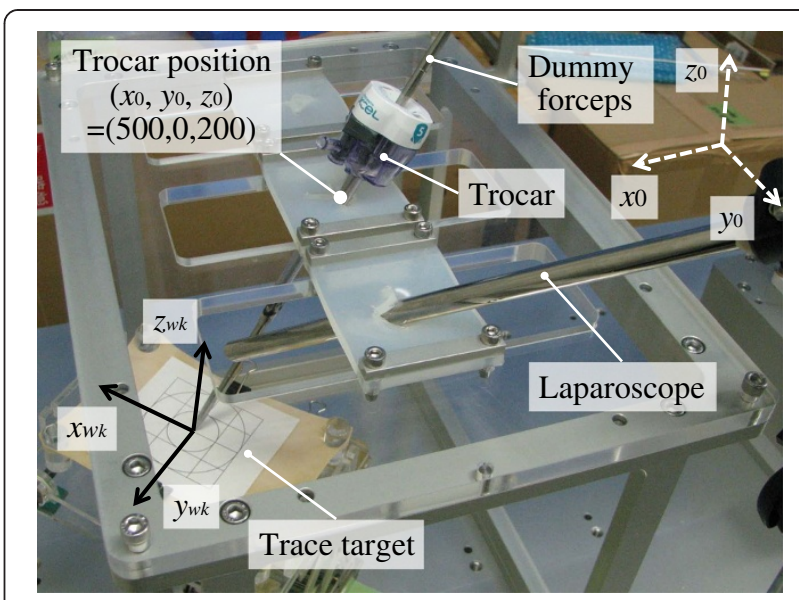

Figure 12 Configuration of trace task.

arm were prepared. One was conventional direct operation of the master arm with a pen-shape device. The other was a master forceps operation of the master arm with a dummy forceps and a virtual trocar. The master arm with the dummy forceps is shown in Figure 6. The master arm with the dummy forceps and the virtual trocar can demonstrate good operating conditions for laparoscopic surgery by maintaining a comfortable position and posture for the surgeon.

Figure 7 shows the joint configuration of the master arm with the dummy forceps and the virtual trocar. The desired point of the slave arm was acquired by the relative position of the origin of the wrist joint coordinate systems of the PHANTOM Omni. A foot switch changed the on-off control of the master-slave mode.

The control system of the slave arm was developed and executed in real time on the RTLinux operating system.

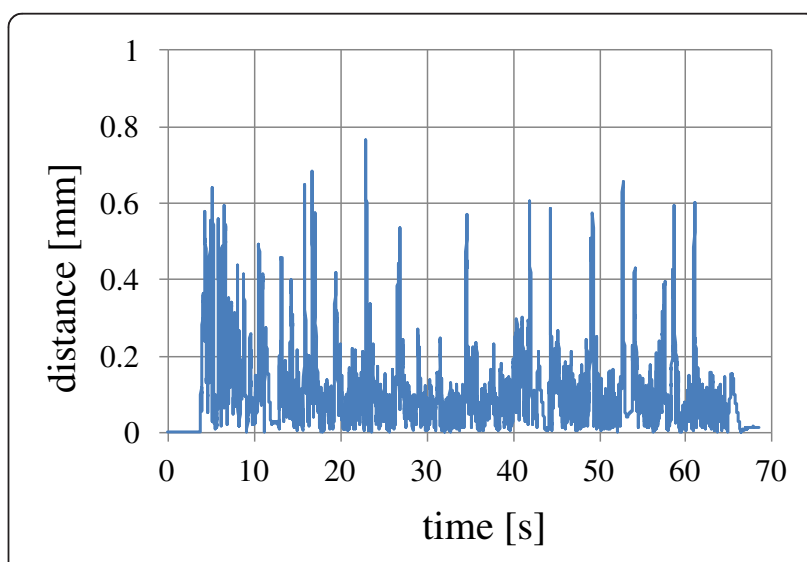

Figure 13 Distance between trocar point and forceps axis while trace task is calculated from joint angles. 


\section{Results and discussion}

The insert motion, remove motion, and master-slave motion were performed by the evaluation experimental system.

Figure 8 shows the overview of the insert motion from the non-trocar mode to trocar mode via the transitional mode. Figures 9 and 10 show the trajectories of the dummy forceps tip for the insert motions from the posture adjustment, insert, and master-slave motions. These figures show the dummy forceps tip trajectories calculated by the forward kinematics from the joint angles based on the base coordinate system in the XY and XZ planes. The trocar position on the base coordinate system is $\left(\mathrm{x}_{0}, \mathrm{y}_{0}, \mathrm{z}_{0}\right)=(500,0,200)$.

Figure 9 shows 3 types of posture adjustment motions by point-to-point, jog, and direct teach motions using the 4th and 5th axes from the same start positions. After the posture adjustment motions, the dummy forceps began the insert motion from same point and passed through the trocar. Furthermore, after the insert motion, the master-slave motions were achieved by the master arm with the dummy forceps and the virtual trocar.

Figure 10 shows the posture adjustment motions by point-to-point motion using the 4th and 5th axes beginning from 3 different positions. After the posture adjustment motions from 3 different starting positions, the dummy forceps began the insert motion and passed through the trocar, and the master-slave motions were achieved by the master arm.

Figure 11 shows the dummy forceps tip trajectories of the slave manipulator when the operator traces the target by the master arm, as shown in Figure 12. Figure 13 shows the calculated distance from the joint angles between the trocar point and forceps axis under the trace task by the master slave motion. The maximal error, which is the maximal distance, was less than $1 \mathrm{~mm}$.

The evaluation experimental system confirmed that the operator can carry out the master-slave motion under the trocar mode through the transition mode that consists of the posture adjustment and insert/remove motions.

\section{Conclusions}

To enable smooth operations when the motion of the trocar is restricted because of the use of a 6-axis vertical articulated robot for the slave arm, a manipulating mode of the slave arm consisting of 4 basic motion modes and 3 control modes has been proposed. Especially, the posture adjustment motion, which is the motion to adjust the forceps axis to the direction of the trocar from any position and posture outside the patient's abdominal cavity, is achieved by movement only in the 4th and 5th axes of the slave arm. The state transition between each control mode has also been demonstrated. Furthermore, the posture adjustment motion and the insert motion under the transitional mode and the master-slave motion under the trocar mode have been verified by evaluation experiments of the master-slave manipulator system including a 6-axis vertical articulated robot in order to confirm the effectiveness of the proposed methods.

Future work for clinical use will include hardware and software development, such as construction of an articulated forceps, systematization of a multi-slave-arm system, planning of a working space, developing a control method in the vicinity of the singular point, and verifying the operability and safety of the system.

\section{Abbreviations}

RCM: Remote center of motion; DOF: Degrees of freedom.

\section{Competing interests}

The author declares that he has no competing interests.

Received: 4 October 2014 Accepted: 26 November 2014

Published online: 09 December 2014

\section{References}

1. Japan Society for Endoscopic Surgery (2012) 11th Nationwide survey of endoscopic surgery in Japan. J Jpn Soc Endosc Surg 17(5):572-694 (in Japanese)

2. Intuitive Surgical, Inc the da Vinci® Surgical System. http://www intuitivesurgical.com/, accessed 9 Sep 2014

3. Guthart GS, Salisbury JK (2000) The intuitive telesurgery system: overview and application. In: Proc. IEEE international conference on robotics and automation, pp 618-621

4. Mitsuishi M, Arata J, Tanaka K, Miyamoto M, Yoshidome T, Iwata S, Hashizume M, Warisawa S (2003) Development of a remote minimally-invasive surgical system with operational environment transmission capability. In: Proc. IEEE international conference on robotics and automation., pp 2663-2670

5. Tadano K, Kawashima K, Kojima K, Tanaka N (2010) Development of a pneumatic surgical manipulator IBIS IV. J of Robotics and Mechatronics 22(2):179-188

6. Matusmoto Y, Katsura S, Ohnishi K (2003) Bilateral control with the virtual supporting point for minimally invasive surgery. In: Proc. Technical Meeting on Industrial Instrumentation and Control, the Institute of Electrical Engineers of Japan IIC-03-33. pp 93-97 (in Japanese)

7. Mayer H, Nagy I, Knoll A, Schirmbeck EU, Bauernschmitt R (2004) The Endo [PA]R system for minimally invasive robotic surgery. In: Proc. IEEE international conference on robotics and automation., pp 3637-3642

8. Tobergte A, Konietschke R, Hirzinger G (2009) Planning and control of a teleoperation system for research in minimally invasive robotic surgery. In: Proc. IEEE international conference on robotics and automation., pp 2098-2105

9. Jinno M, Sunaoshi T, Miyagawa T, Hato T, Matsuhira N, Morikawa Y, Ozawa S, Kitajima M (2006) Development of robotic forceps for laparoscopic surgery. J of Robotics and Mechatronics 18(3):249-254

10. Konietschke R, Hagn U, Nickl M, Jorg S, Tobergte A, Passig G, Seibold U, Le-Tien L, Kubler B, Groger M, Frohlich F, Rink C, Albu-Schaffer A, Grebenstein M, Ortmaier T, Hirzinger G (2009) The DLR MiroSurge - A robotic system for surgery. In: Proc. IEEE international conference on robotics and automation, pp 1147-1148

doi:10.1186/s40648-014-0023-6

Cite this article as: Jinno: Master-slave manipulator for laparoscopic surgery using a 6-axis vertical articulated robot. $R O B O M E C H$ Journal 2014 1:23. 